\title{
Interactive multidimensional programming models for locational decisions
}

\author{
Peter NIJKAMP \\ Department of Economics, Free University, Amsterdam, The \\ Netherlands
}

Jaap SPRONK

Department of Economics, Erasmus University, Rotterdam, The Netherlands

Received May 1979

Revised November 1979
The stringent assumption of a single cost objective will be relaxed by introducing multiple objective functions. This gives rise to a multiobjective optimization (or multidimensional programming) framework (see Section 2). The use of (interactive) multidimensional programming models for the Weber problem will be exposed in Section 3.

The unrealistic assumption of a homogeneous space will be discussed in Section 4. A brief evaluation in Section 5 will conclude this paper.

\section{Interactive multidimensional programming models}

tion theory in two directions. First, the usual assumption of a single cost function will be abandoned by introducing multiple objectives. This gives rise to a multidimensional programming framework for the traditional location models. The paper provides a solution algorithm for the lat ter problem.

Next, the assumption of a uniform space will be tackled by taking account of discrete candidate-lucations. This problem can be solved by means of an adjusted multiciteria andlysis.

The solution algorithms for both extensions are based on an interactive strategy, so that the decision-maker may identify the most favourable location in a finite number of steps.

Key words: Multiple objective, Weber problem, interactive programming

\section{Introduction}

Until recently the Weberian approach, characterized by cost minimization, fixed technical coefficients and a homogeneous space, has dominated the history of the location analysis.

Several severe limitations of the Weber model have been tackled during the last decade. For example, the multi-facilities problem and that of fixed technical coefficients.

The limitations associated with the assumptions of a single uniform cost function and a homogeneous area, however, have received less attention. The present paper will be devoted to overcoming these limitations.

c C North-Holland Publishing Company

European Journal of Operational Research 6 (1981) 220-223
It is an evident fact that the locational decisions for facilities (in both the private and the public sphere) are seldom based exclusively on monetary cost objectives, because

(1) many aspects of investment decisions cannot be translated into a common monetary denominator (risk, e.g.) and

(2) locational decisions usually give rise to external spillover effects or intangibies which can hardly be in zorporated in the traditional cost mechanism (enviiunmental pollution, e.g.).

Consequently, most loc: ional decisions have to be based on a multiplicity of objective functions, for example cosí minimization, risk minimization, maximization of accessibility, minimization of environmental decay, etc. In formal terms, such a multidimensional programmins problem can be formalized as:

$\min \varphi(z)$,

where $\varphi$ is a $I \times 1$ vector of different objective functions $\varphi_{i}$ and $z$ a vector of decision arguments. Clearly, the values of $z$ are constrained by a feasible arec $K$, i.e., $z \in K$

Normally, the various objectives are mutually conflicting, so that a minimization of the one objective precludes the attainment of a minimum value for the other objectives. In the practice of decision-making, normally a compromise between various extreme options is determined in order to reconcile to a certain extent the conflicting objectives (see among 
others Bell et al. [1], Blair [3], Cohon [4]. Haimes et al. [10], Nijkamp [15], Starr and Zeleny [20], Thiriez and Zionts [21] and Zeleny [24]). The identification of such a compromise solution between conflicting options is normally based on the notion of efficient solutions (Pareto solutions). A vector $z \in K$ is an efficient solution if no other feasible solution $z^{0} \in K$ does exist such that:

$\varphi_{i}\left(z^{0}\right) \leqslant \varphi_{i}(z), \quad i=1, \ldots, l$

and

$\varphi_{i}\left(z^{0}\right) \neq \varphi_{i}(z) \quad$ for at least one $i$

This is a first (necessary) demarcation criterion for identifying compromise solutions in multiobjective programming models, but it is obviously not sufficient: the set of efficient solutions is very large.

As indicated above, the specification of trade-offs is fraught with difficulties. Some approaches to gauge trade-offs (relative priorities) for conflicting objectives are an a posteriori revealed preference approach or an a priori inquiry into the decision-maker's preference structure (based on questionnaires, e.g.). In many practical decision problems, however, these approaches fail to provide relevant or reliable irformation.

Therefore, recently much attention has been paid to so-called interactive multiobjective optimization models ${ }^{1}$. The common feature of the majority of these interactive models is that they aim at identifying a compromise solution (or sometimes a subset of most preferred solutions) from the set of efficient solutions by means of a stepwise communication process between the analyst and the decision-maker. The analyst provides the decision-maker with information about trade-offs between conflicting solutions, while he also shows him in a stepwise manner one or more compromise solutions. Then the decision-maker has to express his preferences concerning these provisional compromise solutions, for example, by rejecting nonsatisfactory solutions. Next, the analyst may incorporate this information in his optimization process and suggest a new compromise solution to the decision-maker. This procedure is repeated, until a final satisfactory compromise solution is achieved. The advantages of such a procedure are: it provides information to the decision-maker in a stepwise way, it

1 See among others Benayoun et al. [2], Geoffricn et al. [9], Monarchi et al. [12], Nijkamp and Rietveld [14], Nijkamp en Spronk [16], Pollack [18], Walker [22] and Wallenius [23]. stimulates an active role of the decision-maker, and it avoids the prior specification of trade-offs. The abovementioned interactive approach will now be applied to a multiobjective Weber problem.

\section{An interactive multidimensional programming model for continuous location problems}

The general aim of the traditional Weber model is to find the minimum cost location for a firm or a facility, given the location of the input sources and of the markets. Normally, uniform transportation costs over a homogeneous area and fixed technological coefficients are assumed.

If the (known) production volume of the firm concerned is denoted by $q$ and if the successive points of inputs (raw materials, energy, etc.) and outputs (final products, iniermediate deliveries etc.) are numbered as $1, \ldots, n, \ldots . N$, the transportation costs $T_{n}$ from the as yet unknown optimal location of the firn to an arbitrary point $n$ are equal to:

$T_{n}(x, v)=c_{n} d_{n}(x, y)$

where $c_{n}=$ cost per unit of distance between point $n$ and the facility, given the volume of production and $d_{n}(x, y)=$ distance between point $n$ located at $\left(x_{n}, y_{n}\right)$ and a facility located at $(x, y)$.

The Euclidean distance $d_{n}$ is given by:

$d_{n}(x, y)=\left\{\left(x_{n}-x\right)^{2}+\left(y_{n}-y\right)^{2}\right\}^{1 / 2} \quad \forall n$.

Then the total transportation costs $T(x, y)$ to all $N$ places are:

$T(x, y)=\sum_{n=1}^{N} T_{n}(x, y)$.

If we assume that the firm has I linear objective functions $\varphi_{1}, \ldots, \varphi_{1}$ for its locational decisions, the general specification of the $i$ th objective function is:

$\min \varphi_{i}(x, y)=\sum_{n=1}^{N} T_{n i}(x, y)$.

For each objective function $\varphi_{i}$, the minimum value $r_{i}^{0}$ can be calculated by means of a standard solution algorithm which consists oi an iterative numerical approximation of the optimum (see, for example, Kuhn and Kuenne [11] and Cooper [5]).

These solutions are then included in the vector $\varphi^{0}$, called the ideal point. In a similar way, for each objec- 
tive function $\varphi_{i}$ the minimizing co-ordinates $\left(x_{i}, y_{i}\right)$ can be determined. Because the various objective functions are normally conflicting, the $I$ pairs of co-ordinates $\left(x_{1}, y_{1}\right) \ldots,\left(x_{I}, y_{I}\right)$ do not coincide.

Normally, the trade-offs associated with the objective functions are not specified in advance by the decision-maker, so that it will be useful to proceed via the following interactive procedure:

- calculate the ideal point $\varphi^{0}$ with arguments $\varphi_{1}^{0}, \ldots$, $\varphi_{I}^{0}$;

- calculate the compromise point $\varphi$ with arguments $\hat{\varphi}_{1}, \ldots, \hat{\varphi}_{I}$ on the basis of the gravity point of the co-ordinates $\left(x_{1}, y_{1}\right), \ldots,\left(x_{I}, y_{I}\right)$ associated with the ideal point ${ }^{2}$;

- ask the decision-maker to express his opinion about the compromise solutions $\hat{\varphi}_{1}, \ldots, \hat{\varphi}_{I}$ such that he may identify an element $\hat{\varphi}_{i}^{\prime}$ of the compromise solutions which he judges as least satisfactory. This gives rise to the foilowing side-condition:

$\varphi_{i}^{\prime}<\hat{\varphi}_{i}^{\prime}$;

- include (8) as a constraint in the original multiobjective location model, so that one obtains:

$$
\min \varphi_{i} \quad \forall i, \quad \varphi_{i}^{\prime}<\hat{\varphi}_{i}^{\prime} .
$$

Then the procedure has to be repeated, until finally a satisfactory compromise solution has been found (see for a discussion of convergence properties Fandel [8]).

There is only one problem left, viz. the solution of (9). This constrained nonlinear programming model cannot be solved directly by means of the algorithm developed for the orginal unconstrained Weber problem.

Two ways are open to solve the nonlinear multiobjective programming model implied by (9). The first way is to rewrite (9) as a general geometric programming model (see among others Duffin et al. [7] and Nijkamp [13]). By defining the elements from the distance relationship $d_{n i}$ included in (7) successively as:

$d_{n i}=z_{n i}^{1 / 2}$,

$z_{n i}=\dot{x}_{n i}^{2}+\dot{y}_{n i}^{2}$

and

$\dot{x}_{n i}=x_{i}-x_{n i}, \quad \dot{y}_{n i}=y_{i}-y_{n i}$

it is easily seen that $\varphi_{i}$ has the standard format of the

2 An alternative procedure may be to minimize the distance between the ideal point and the efficiency frontier (see [14]). objective function of a geometric programming model. The same holds true for the constraint from (9). Therefore, in principle, one may solve the single-objective version of ( 9 ) by means of a standard algorithm for geometric programming models (see for a survey Rijckaert and Martens [19]). The seperate singleobjective solutions can then be used to calculate the new compromise points etc.

An alternative way of treating (9) is to use a heuristic algorithm which may be less elegant in itself, but which is closely linked to the single-objective Weber algorithm. Then, the following steps have to be undertaken:

(1) Calculate the unconstrained minima of each objective function $\varphi_{i}$ and check whether the constraint specified in ( 8 ) is effective. If not, the minimum value of the objective function concerned can be included in the ideal point. Otherwise, proceed to (2).

(2) If the minimization of a certain $\varphi_{i}$ leads to certain effective constraint, $\varphi_{i}^{\prime}$, construct the following Lagrange expression with a multiplier $\lambda$ :

$L=\varphi_{i}-\lambda\left(\varphi_{i}^{\prime}-\hat{\varphi}_{i}^{\prime}\right)$.

(3) Calculate the first-order derivatives with respect to $x$ and $y$ :

$\frac{\delta L}{\delta x}=\frac{\delta \varphi_{i}}{\delta x}-\lambda \frac{\delta \varphi_{i}^{\prime}}{\delta x}=0$,

$\frac{\delta L}{\delta y}=\frac{\delta \varphi_{i}}{\delta y}-\lambda \frac{\delta \varphi_{i}^{\prime}}{\delta y}=0$,

$\varphi_{i}^{\prime}=\hat{\varphi}_{i}^{\prime}$.

The first two equations of (14) would have a unique solution, when $\lambda$ would have a unique solution, when $\lambda$ would be known, because in that case their structure would formally be equal to the first order conditions of the standard single-objective Weber problem.

Therefore, the following trial-and-error procedure is reasonable:

(4) Substitute an arbitrary initial solution of $\lambda$ into (14) and solve the corresponding values of $x$ and $y$ on the basis of the two first equations. Substitute these values into the last constraint of (14) and check whether these values are correct. If not, adjust $\lambda$ by means of a grid research proceciure until the last constraint is satisfied (see for such a grid procedure also Paelinck and Nijkamp [17]).

This procedure can be carried out for each active constraint, so that after a series of stages (leading to a reduction of the feasible area) a satisfactory compro- 
mise point can be found. When the step sizes of the grid search procedure are not extremely large, a unique solution can normally be found in a limited number of steps. In this way a convergence is usually guaranteed.

\section{An interactive multidimensional programming model for discrete location problems}

In the previous sections a homogeneous area was assumed. In reality, this assumption is often violated, because decision-nakers normally make a choice out of a distinct number of candidate-locations.

This discrete problem cannot be solved by means of the traditional Weber alyorithms. Recently, however, these kinds of distinct evaluation problems have been studied quite extensively in discrete multicriteria analysis.

Discrete interactive procedures which run parallel to the above-mentioned continuous interactive procedure have shown their practical relevance in reality (see Van Delft and Nijkamp [6], and Nijkamp and Spronk [16]). Moreover, they can be adjusted in several ways, for example, by including multiple decisionmakers, multiple facilities, and soft (ordinal or qualitative) information.

\section{Conclusion}

The locational decision problems discussed in the previous sections were based on the traditional Weber analysis. Their aim was to tackle some of the severe limitations of the Weber approach. A multiobjective programming framework appeared to provide a signicant extension of the traditional restrictive Weber analysis. It also appeared that discrete multicriteria analysis was another useful tool for locational analysis, especially for distinct location problems. The general conclusion is that, in particular the interactive variants of multiobjective optimization models offer many opportunities for a more satisfactory and modern locational analysis.

\section{References}

[1] D.E. Bell, R.L. Keeney and H. Raiffa, Conflicting Objectives in Decisions (Wiley, New York, 1977).

[2] R. Benayoun, J. de Montgolfier, J. Tergny and O. Larichev, Linear programming with multiple objective functions: step method (STEM), Math. Programming 1 (1971) 366-375.

[3] P.D. Blair, Multiobjective Regional Energy Planning (Nijhoff, The Hague, 1979).

[4] J.L. Cohon, Multiobjective Programming and Planning (Academic Press, New York, 1978).

[5] L. Cooper, Solution of generalized locational equilibrium models, J. Regional Sci. 7 (1) (1967) 1-18.

[6] A. van Delft and P. Nijkamp, Multicriteria Analysis and Regional Decision-Making (Nijhoff, The Hague/ Boston, 1977).

[7] R.J. Duffin, E.L. Peterson, and C. Zener, Geometric Programming (Wiley, New York, 1967).

[8] G. Fandel, Optimale Entscheidung bei Mehrfacher Zielsetzung (Springer, Berlin, 1972).

[9] A.M. Geoffrion, J.S. Dyer, and A. Feinberg, An interactive approach for multicriterion optimization with an application to the operation of an academic department, Management Sci. 19 (1972) 357-368.

[10] Y.Y. Haimes, W.A. Hall and H.T. Freedman, Multiobjective Optimization in Water Resource Systems (Elsevier, Amsterdam, 1975).

[11] H.W. Kuhn and R.E. Kuenne, An efficient algorithm for the numerical solution of the generalized Weber problem in spatial economics, J. Regional Sci. 4 (2) (1962) 21-33.

[12] D.E. Monarchi, J.E. Weber and L. Duckstein, An interactive multiple objective decision-making aid using norlinear goal programming, in: Multiple Criteria Decision-Making (Springer, Berlin, 1976) 235-253.

[13] P. Nijkamp, Planning of Industrial Complexes by means of Geometric Programming (Teakfield, Farnborough, 1972).

[14] P. Nijkamp and P. Rietveld, Conflicting social priorities and compromise social decisions, in: I. Cullen, Ed., Analysis and Decision in Regional Planning (Pion, London 1979) 153-177.

[15] P. Nijkamp, Multidimensional Spatial Data and Decision Analysis (Wiley, New York/London, 1979).

[16] P. Nijkamp and J. Spronk, Analy sis of production and location decisions by means of multicriteria analy sis, Eng. Process Economics 4 (1979) 285-302.

[17] J.H.P. Paelinck and P. Nijkamp, Operational Theory and Method of Reginonal Economics (Saxon House, Farnborough, 1976).

[18] M. Pollack, Interactive models in operations research an introduction and some future research directions, Comput. \& Operations Res. 3(4) (1976) 305-312.

[19] M.J. Rijckaert and X.M. Martens, Comparison of generalized geometric programming algorithms, J. Optimization Theory Appl. 26 (2) (1978) 205-272.

[20] M.K. Starr and M. Zeleny, Editors, Multiple Criteria Decision Making (North-Holland, Amsterdarn, 1977).

[21] H. Thiriez and S. Zionts, Editors, Multiple Criteria Decision Making (Springer, Berlin, 1976).

[22] J. Walker, An interactive method as an aid in solving bicriterion mathematical programming problems, $\mathbf{J}$. Operational Res. Soc. 29(9) 915-922.

[23] J. Wallenius, Iriteractive Multiple Criteria Decision Methods (Helsinki School of Economics, Helsinki, 1975).

[24] M.Zeleny, Editor, Multiple Criteria Decision Making (Springer, Berlin, 1976). 